\title{
AVALIAÇÃO DOS MÉTODOS DE GRANT, VORA \& WEEKS E DOS MÍNIMOS QUADRADOS NA DETERMINAÇÃO DO VALOR INCREMENTAL DO MERCADO DE CARBONO NOS PROJETOS DE GERAÇÃO DE ENERGIA ELÉTRICA NO BRASIL
}

\author{
Fabio Rodrigo Siqueira Batista ${ }^{1 *}$, José Paulo Teixeira ${ }^{2}$, \\ Tara Keshar Nanda Baidya² e Albert Cordeiro Geber de Melo $^{3}$
}

Recebido em dezembro 2008 / Aceito em maio 2010

\begin{abstract}
The objective of this paper is to evaluate the robustness of the Grant, Vora \& Weeks and Least Square Monte Carlo Methods when used to evaluate renewable generation projects developed according to the rules of the Kyoto Protocol Clean Development Mechanism. The proposed methodology makes use of the NEWAVE model in order to generate futures dispatch sequences for all generators connected to the Brazilian grid. After that, based on the methodology ACM0002, the uncertainty associated to the time evolution of the project's baseline is considered. Finally, the carbon market' incremental payoff is estimated using the Real Options Approach. In order to do that, the numerical methods previously mentioned are used under the assumption that the behavior of the carbon price is random and that the price follows a Geometric Brownian Motion.
\end{abstract}

Keywords: real options, carbon market, renewable energy sources.

RESUMO. Este trabalho tem por objetivo avaliar a robustez dos métodos de Grant, Vora \& Weeks e dos Mínimos Quadrados quando aplicados na avaliação de projetos de geração de energia elétrica a partir de fontes renováveis desenvolvidos no âmbito do Mecanismo de Desenvolvimento Limpo do Protocolo de Quioto. Em primeiro lugar, a metodologia proposta consiste em utilizar o modelo NEWAVE para estabelecer cenários hidrológicos de despacho para as usinas conectadas ao sistema interligado nacional. Em seguida, baseado no escopo da metodologia ACM0002, considera-se a incerteza associada à evolução temporal da linha de base do projeto. Finalmente, o valor incremental do mercado de carbono é estimado utilizando-se a Teoria das Opções Reais. Para tanto os métodos numéricos citados anteriormente são utilizados sob a premissa de que o preço do crédito de carbono se comporta de forma aleatória segundo as premissas de um Movimento Geométrico Browniano.

Palavras-chave: opções reais, mercado de carbono, fontes renováveis de energia.

\footnotetext{
*Autor correspondente

${ }^{1}$ PUC-Rio - DEI/CEPEL. - E-mail: fabiorsb@hotmail.com

${ }^{2}$ PUC-Rio - DEI.

${ }^{3}$ CEPEL / UERJ.
} 


\section{INTRODUÇÃO}

Atualmente o método mais difundido e aplicado para a análise de investimentos é o Valor Presente Líquido (VPL). Entretanto, segundo Dixit \& Pindyck (1994), o VPL pode ser considerado um método ortodoxo, excessivamente rígido para o ambiente coorporativo. Isso ocorre porque este método sugere que o investimento deva ser realizado agora ou nunca, ignorando os benefícios da espera por novas informações. Implicitamente, o VPL presume que o gerenciamento de projetos é passivo, ou seja, que todas as decisões são inexoravelmente levadas adiante, como se o investidor não dispusesse de flexibilidade para rever os planos originais.

A maioria das decisões de investimento tem em comum três características importantes: a sua irreversibilidade, a incerteza sobre os ganhos futuros, e a liberdade de ação no tempo. O fato é que a teoria ortodoxa não reconhece estas características (vide Dixit \& Pindyck, 1994). No entanto, é justamente a interação entre elas que determina a regra ótima para o investimento.

É importante observar que a empresa que possui uma oportunidade de investimento, na verdade possui o direito, mas não a obrigação de realizar esse investimento. Uma vez que o investimento é realizado, a empresa anulará qualquer possibilidade de espera, o que a impossibilita de agir no sentido de reverter uma situação desfavorável no futuro. Sendo assim, nota-se que a decisão de investir implica em um custo de oportunidade para a empresa, o qual deve ser incluído junto aos custos de investimento no projeto.

Desta forma, a regra convencional do VPL deve ser modificada de tal forma a sinalizar que o investimento será ótimo apenas quando o valor presente dos ganhos for maior que o valor presente dos custos, incluindo o custo de oportunidade por abrir mão dos benefícios da espera. Este novo valor é denominado VPL Expandido, o qual reúne o valor das opções operacionais e estratégicas resultantes do gerenciamento dinâmico do projeto. Estas opções, por serem baseadas em ativos reais, são conhecidas como opções reais.

Apesar da crescente utilização da Teoria das Opções Reais (TOR) para a avaliação dos mais diversos tipos de investimentos, por exemplo, na avaliação de reservas de recursos naturais (vide Brennan \& Schwartz, 1985; Siegel et al., 1987; Dias, 1996 e 2005; etc.), apenas nos últimos anos a aplicação desta teoria começou a penetrar a indústria da eletricidade. Dentre as suas principais aplicações ao setor elétrico brasileiro, destacam-se os trabalhos de Castro (2000) e Gomes (2002). Em ambos os casos esta teoria foi utilizada na avaliação de investimentos em geração termelétrica no Brasil. No âmbito internacional, trabalhos mais recentes, como os de Kumbaroglu et al. (2004) e Siddiqui et al. (2005), aplicam a Teoria das Opções Reais para avaliar investimentos na geração de energia elétrica com base em fontes renováveis, tais como as fontes eólicas, geotérmicas, solares, entre outras.

Mais recentemente, a TOR também começou a ser empregada no recém criado mercado de carbono, entretanto, até o momento, as principais aplicações têm se concentrado nas atividades de florestamento e reflorestamento (vide Baran, 2005 e Ruolz, 2001). Em outros casos, o método mais empregado ainda tem sido o Valor Presente Líquido (vide Barros, 2006).

Neste contexto, um dos objetivos deste trabalho é utilizar a Teoria das Opções Reais como um dos pilares de uma metodologia que visa estimar o valor incremental do mercado de carbono 
para empreendimentos brasileiros de geração de energia elétrica a partir de fontes renováveis. Para tanto, considera-se que este tipo de projeto traz consigo a flexibilidade de ser registrado no Comitê Executivo das Nações Unidas, e comercializar os Créditos de Carbono (também denominados Reduções Certificadas de Emissões - RCEs) que venham a ser gerados pela sua atividade.

Para avaliar corretamente a flexibilidade anteriormente descrita, é necessário perceber a sua forte analogia com as opções de compra disponíveis no mercado financeiro. Neste caso, nota-se que o valor presente da receita obtida a partir da venda das RCEs pode ser entendido como o valor do ativo objeto de uma opção financeira. Da mesma forma, os custos de transação, necessários para que se efetue o registro do projeto no Comitê Executivo, podem ser entendidos como o seu preço de exercício. Além disso, o prazo disponível para que o investidor registre o projeto MDL pode ser entendido como o tempo de vida da opção financeira. Finalmente, a volatilidade do preço da RCE e os fluxos anuais provenientes da sua venda podem ser entendidos, respectivamente, como a volatilidade e o dividendo do ativo objeto da opção financeira.

Enfim, fica claro que as mesmas técnicas empregadas para se avaliar opções financeiras podem ser utilizadas para avaliar opções sobre ativos reais. Em resumo, destacam-se como os principais objetivos deste trabalho:

- desenvolver um arcabouço metodológico que permita a estimação do valor incremental do mercado de carbono nos projetos de geração de energia elétrica conectados à sistemas hidrotérmicos interligados, tal como o sistema brasileiro;

- considerar a aleatoriedade nos preços das Reduções Certificadas de Emissões associadas ao desenvolvimento de projetos MDL de geração de energia elétrica a partir de fontes renováveis;

- verificar a robustez e a convergência de determinados métodos numéricos, inicialmente desenvolvidos para a avaliação de opções, na determinação do valor incremental do mercado de carbono para projetos com as características descritas anteriormente.

Em particular, os métodos analisados serão os métodos de Grant, Vora \& Weeks (1996) e de Longstaff \& Schwartz (2001). Em ambos os casos os resultados obtidos pela utilização do método binomial (1979) serão utilizados como benchmark das análises.

\section{MÉTODOS PARA A AVALIAÇÃO DE OPÇÕES FINANCEIRAS}

Nesta seção os métodos Binomial, de Grant, Vora \& Weeks, e de Longstaff \& Schwartz serão descritos detalhadamente. Conforme descrito na seção anterior, neste trabalho a convergência destes métodos será testada no sentido de viabilizar a sua utilização para avaliar a oportunidade que determinados projetos de geração de energia elétrica possuem para produzir e comercializar RCEs.

\subsection{Modelo binomial}

Considerando $T$ o prazo até o vencimento da opção, a aplicação do método binomial pressupõe que o intervalo $T$ deve ser subdividido em $N$ intervalos de extensão $\Delta t=T / N$. Para cada 
intervalo $\Delta t$, considera-se que o preço do ativo objeto da opção $(S)$ está sujeito a apenas dois tipos de variações: uma variação positiva de intensidade $u$, ou seja, $S_{t+\Delta t}=S_{t} \cdot u$, ou uma variação negativa de intensidade $d$, ou seja, $S_{t+\Delta t}=S_{t} \cdot d$. Nesta formulação os parâmetros $u$ e $d$ são respectivamente determinados por $e^{\sigma \sqrt{\Delta t}}$ e $e^{-\sigma \sqrt{\Delta t}}$, onde $\sigma$ representa a volatilidade do ativo objeto da opção.

Cabe destacar que o método binomial considera que os movimentos de alta e de baixa dos preços do ativo objeto estão associados à probabilidades neutras ao risco, respectivamente denominadas por $q$ e $(1-q)$. Segundo Cox, Ross \& Rubinstein (1979), a probabilidade $q$ pode ser determinada pela seguinte equação:

$$
q=\{\exp [(r-\delta) \cdot \Delta t]-d\} /(u-d)
$$

onde $r$ representa a taxa de juros livre de risco e $\delta$ representa o dividend yield do ativo objeto da opção. Com o objetivo de determinar o valor de uma opção americana de compra, uma rotina de programação dinâmica é iniciada a partir da sua data de vencimento $(T)$. Neste instante, o valor da opção deve ser estabelecido por meio da seguinte equação:

$$
\left.C_{T}^{i}\left(S_{T}^{i}\right)=\max \left(S_{T}^{i}-X, 0\right)\right) \quad \forall i
$$

onde $i$ representa o número de preços (ou nós) da árvore binomial no instante $T, T$ representa a maturidade da opção, e $X$ representa o seu preço de exercício.

Caminhando-se recursivamente, em cada nó intermediário representado no instante $t=T-\Delta t$, o valor da opção deve ser obtido comparando-se o valor de exercício imediato $\left(I_{t}\right)$ com o seu valor de continuação $\left(F_{t}\right)$, ou seja, o valor de se manter a opção viva até o próximo instante. A equação 3 representa esta comparação.

$$
C_{t}^{i}\left(S_{t}^{i}\right)=\max \left\{I_{t}^{i}, F_{t}^{i}\right\} \quad \forall i
$$

onde

$$
I_{t}^{i}=\max \left(S_{t}^{i}-X, 0\right)
$$

$\mathrm{e}$

$$
F_{t}^{i}=e^{-r \cdot \Delta t}\left(q \cdot C_{t+\Delta t}\left(u \cdot S_{t}^{i}\right)+(1-q) \cdot C_{t+\Delta t}\left(d \cdot S_{t}^{i}\right)\right) .
$$

Nas equações 3, 4 e 5, nota-se que $i$ representa o número de nós da árvore binomial no instante $t$ considerado, sendo os parâmetros $q, u$ e $d$ determinados conforme definido anteriormente. Devese seguir trabalhando recursivamente até o instante inicial da análise $(t=0)$, quando o valor da opção é determinado empregando-se o mesmo raciocínio, descrito anteriormente, para todos os nós intermediários representados pela árvore binomial.

Finalmente, ressalta-se que a grande vantagem deste modelo é a sua capacidade em avaliar a possibilidade de exercício antecipado das opções americanas, fornecendo uma boa aproximação do seu valor. Por outro lado, o maior problema deste método reside na hipótese de que o preço do ativo objeto é o único fator aleatório a ser considerado na análise, uma vez que a solução do problema se torna computacionalmente inviável à medida que o número de fatores estocásticos aumenta. 


\subsection{Método de Grant, Vora \& Weeks (GVW)}

O princípio básico deste método consiste em identificar, para cada instante anterior ao vencimento da opção, o preço crítico do seu ativo objeto, ou seja, o preço no qual o investidor é indiferente entre exercer ou não a opção naquele momento. Uma vez conhecidos estes valores, argumenta-se que o derivativo americano pode ser avaliado da mesma forma que um derivativo europeu, ou seja, calculando-se a média aritmética de valores previamente simulados.

Nota-se ainda que a partir do conjunto de preços críticos $\left(S_{t}^{*}\right)$ de uma opção, duas regiões podem ser definidas: a região de exercício antecipado, onde exercer a opção é a decisão ótima, e a região de continuação, onde a melhor estratégia é esperar até o próximo instante para tomar uma nova decisão. A curva de indiferença entre essas regiões é denominada Fronteira de Exercício Ótimo, ou Curva de Gatilho do derivativo.

Considerando uma opção americana de compra que possa ser exercida em qualquer instante $t \in[0, T]$, com preço de exercício $X$, e com preço do ativo no instante $t$ representado por $S_{t}$, segundo GVW o valor desta opção $\left(C_{t}\right)$ pode ser determinado segundo a equação 6 mostrada a seguir:

$$
C_{t}\left(S_{t}, X\right)=\max \left\{I_{t}, F_{t}\right\}
$$

onde

$$
I_{t}=\max \left\{S_{t}-X, 0\right\}
$$

e

$$
F_{t}=e^{-r \cdot \Delta t} E_{t}\left[C_{t+\Delta t}\left(S_{t+\Delta t}, X\right)\right]
$$

Esta equação é similar à equação 3 definida na seção anterior. Novamente, o primeiro termo do operador de maximização representa o valor do exercício imediato da opção, enquanto que o segundo representa o seu valor de continuação. Cabe destacar que para determinar o valor de continuação pelo método de GVW, é necessário o conhecimento prévio de todos os preços críticos entre os instantes $t$ e o vencimento da opção.

Uma vez que o preço crítico representa o preço para o qual o valor intrínseco do derivativo é igual ao seu valor de continuação, é possível definir uma condição de contorno para $S_{t}^{*}$ igualando-se as equações 7 e 8 , ou seja:

$$
S_{t}^{*}-X=e^{-r \cdot \Delta t} E_{t}\left[C_{t+\Delta t}\left(S_{t+\Delta t}^{*}, X\right)\right]
$$

A partir desta equação, conclui-se que o valor de $S^{*}$ pode ser facilmente determinado para a data de vencimento do derivativo. Note que, na maturidade, o valor de continuação do derivativo é igual a zero, pois não haverá outra oportunidade para o seu exercício. Sendo assim, a equação 9 pode ser reescrita da seguinte forma:

$$
S_{T}^{*}-X=0
$$

ou seja, $S_{T}^{*}=X$. Uma vez que a determinação de $S_{t}^{*}$ depende do conhecimento prévio de todos os preços críticos nos instantes posteriores a $t$, GVW propõem que a curva de gatilho seja determinada recursivamente, empregando-se a técnica de Programação Dinâmica. 
O processo de otimização tem início no instante anterior ao vencimento da opção, ou seja, em $T-\Delta t$. O portador da opção de compra pode exercê-la imediatamente ou manter a opção "viva" até a sua maturidade. Empregando-se a equação 6, o valor da opção pode ser determinado da seguinte forma:

$$
C_{T-\Delta t}\left(S_{T-\Delta t}, X\right)=\max \left\{I_{T-\Delta t}, e^{-r \cdot \Delta t} E_{T-\Delta t}\left[C_{T}\left(S_{T}, X\right)\right]\right\}
$$

O preço crítico $\left(S_{T-\Delta t}^{*}\right)$ é identificado encontrando-se o valor de $S_{T-\Delta t}$ que satisfaz a condição 9. Assumindo que seja possível identificar $S_{T-\Delta t}^{*}$, a otimização continua identificando-se o valor de $S_{T-2 \Delta t}^{*}$ condicional ao conhecimento de $S_{T-\Delta t}^{*}$ e $S_{T}^{*}$. Por esta lógica, o processo continua até a determinação de $S_{0}^{*}$.

Segundo a condição 9 , determinar o valor de $S_{t}^{*}$ implica em determinar o valor de continuação $(F)$ associado ao instante $t$, entretanto, informações sobre preços futuros ainda não são conhecidas neste instante. Grant, Vora \& Weeks solucionam este problema empregando a técnica de Simulação de Monte Carlo (SMC).

A SMC é iniciada em $T-\Delta t$, adotando-se como condição inicial $S_{T-\Delta t}^{*}=S_{T}^{*}$. Uma vez arbitrado um valor inicial para $S_{T-\Delta t}^{*}$, valores de $S_{T}$ são simulados a fim de se determinar o valor de continuação da opção. Caso a condição 9 não seja satisfeita, o valor de $S_{T-\Delta t}^{*}$ deve ser incrementado e a SMC repetida. Esta rotina deve ser realizada até que a condição 9 seja atendida.

O processo de solução continua, recursivamente, ao longo da vida da opção. Uma vez determinada a curva de gatilho do derivativo, determina-se o valor da opção através de $N$ simulações de Monte Carlo iniciadas em $t=0$. Para tanto, é considerado um preço inicial para o ativo objeto $\left(S_{0}\right)$ dado pelo mercado. O exercício antecipado ocorre no primeiro instante em que o preço do ativo ultrapassa a curva de gatilho. O valor final da opção é então determinado através da média dos valores obtidos para cada trajetória simulada, ou seja,

$$
C_{0}=\frac{1}{N} \sum_{w=1}^{N} e^{-r \cdot \tau}\left[\max \left(S_{\tau}^{w}-X, 0\right)\right] \quad \forall S_{\tau}^{w}>S_{\tau}^{*}
$$

Nesta equação, $\tau$ representa o primeiro instante em que o preço simulado ultrapassa a curva de gatilho.

Deve-se ressaltar que uma das principais vantagens do método de GVW é o fato dele ser independente do número de dimensões do problema, de tal forma que a curva de gatilho irá sempre possuir a mesma dimensão do derivativo o qual se pretende avaliar. Além disso, este método é geral e aplicável a diferentes tipos de opções, ou mesmo a opções com vários parâmetros estocásticos. Por outro lado, a sua grande desvantagem é o seu alto custo computacional, o qual é devido à necessidade da realização de um grande número de Simulações de Monte Carlo.

\subsection{Método dos Mínimos Quadrados (LSM)}

Após analisar o método binomial e o método de GVW, nota-se que a decisão de exercer antecipadamente uma opção americana se baseia, principalmente, na comparação entre o seu valor 
intrínseco e o valor de continuação do derivativo. Conforme descrito nas Seções 2.1 e 2.2, determinar o valor intrínseco de uma opção pode ser considerado uma tarefa pouco complexa, entretanto, uma boa estimativa do seu valor de continuação é mais difícil de ser obtida. Conforme mencionado anteriormente, aplicando-se o método de GVW este processo demanda a realização de um grande número de SMC, o que pode levar a elevados custos computacionais.

Neste sentido, Longstaff \& Schwartz (2001) propuseram uma metodologia que reduz o custo computacional dos métodos de simulação. Comparado ao método de GVW, a principal diferença do método proposto por Longstaff \& Schwartz reside no cálculo do valor de continuação. Enquanto GVW estimam este valor por meio de simulações, Longstaff \& Schwartz propõem que sejam realizadas regressões utilizando informações cross-sectional sobre o preço do ativo financeiro. Este método é denominado Least Square Monte Carlo (Método dos Mínimos Quadrados), ou, simplesmente, LSM.

O primeiro passo do método LSM consiste em definir um número finito de datas onde é possível o exercício antecipado da opção. Desta forma, considerando $T$ o vencimento do derivativo, assume-se que a vida da opção pode ser dividida em $D$ intervalos iguais de tamanho $\Delta t=T / D$. Uma vez simuladas $N$ trajetórias para o preço do ativo objeto, Longstaff \& Schwartz consideram que o valor de continuação pode ser inicialmente definido por meio da seguinte equação:

$$
F(w, t)=E_{Q}\left[\sum_{t_{j}=t+\Delta t}^{T} e^{-r\left(t_{j}-t\right)} \cdot V\left(w, t_{j}, t, T\right) / \Im_{t}\right]
$$

onde $t$ representa um instante qualquer dentro do intervalo $[0, T], w$ representa uma das trajetórias simuladas, $Q$ representa uma medida de probabilidade neutra ao risco e $\Im_{t}$ representa o conjunto de informações disponíveis em $t$. Ainda na equação 12, ressalta-se que $V\left(w, t_{j}, t, T\right)$ representa o fluxo de caixa gerado pelo exercício da opção em qualquer instante $t_{j}>t$. Uma vez que as opções americanas podem ser exercidas apenas uma vez em cada trajetória $w$, cabe ressaltar que, no máximo, existirá um $t_{j}$ tal que $V\left(w, t_{j}, t, T\right)>0$.

Conforme mencionado anteriormente, Longstaff \& Schwartz supõem que o valor de continuação $(F(w, t))$ pode ser melhor estimado por meio de regressões cross-sectional sobre o preço do ativo financeiro. O algoritmo se sustenta na idéia de que $F(w, t)$ pode ser representado por meio de uma combinação linear de funções base $\left(B_{l}\right)$, cujas constantes são determinadas através de uma regressão dos mínimos quadrados. Este raciocínio é representado pela equação 13, onde $S$ representa o preço do ativo objeto da opção e $a_{l}$ representa a constante associada a cada função base $B_{l}$.

$$
F(w, t)=\sum_{l=0}^{\infty} a_{l} B_{l}(S)
$$

A equação 13 considera infinitos termos para o cálculo de $F(w, t)$, entretanto, para fins práticos, essa consideração não é viável computacionalmente. Neste caso, o valor de $F(w, t)$ deve ser aproximado utilizando-se um número $G<\infty$ de funções base, ou seja:

$$
F(w, t) \approx F_{G}(w, t)=\sum_{l=0}^{G} a_{l} B_{l}(S)
$$


A partir da equação 14 o método LSM estima o valor de $F_{G}(w, t)$ regredindo os valores de continuação inicialmente calculados em relação às funções base pré-definidas. Em um dado instante $t$, tal regressão é realizada considerando apenas às trajetórias em que a opção se encontra in-the-money, pois somente para estas trajetórias a decisão de exercício antecipado é relevante.

Uma vez estimado o valor de continuação da opção, a decisão de exercê-la antecipadamente é tomada comparando-se o seu valor intrínseco com o valor de continuação estimado. Assim como no método Binomial e de GVW, o processo iterativo do método LSM é recursivo. O valor da opção $\left(C_{L S}\right)$ é aproximado calculando-se a média aritmética da soma de todos os fluxos de caixa $V\left(w, t_{j}, t, T\right)$ onde o exercício da opção é ótimo, ou seja:

$$
C_{L S}=\frac{1}{N} \sum_{w=1}^{N} \sum_{t_{j}=\Delta t}^{T} e^{-r \cdot t_{j}} V\left(w, t_{j}, 0, T\right)
$$

Ainda sobre o método LSM, fica claro que o relativo baixo custo computacional caracteriza a sua principal vantagem em relação aos demais métodos que envolvem a SMC na precificação de derivativos. Além disso, tal com o método de GVW, destaca-se que este método também permite a avaliação de diferentes tipos de opções, envolvendo diferentes processos estocásticos, ou mesmo opções com diferentes dimensões.

Conforme o apresentado nesta seção, para que uma opção financeira possa ser devidamente avaliada, é necessário representar adequadamente a dinâmica do seu ativo objeto ao longo do tempo. Uma vez que neste trabalho os resultados obtidos pelo modelo binomial definido por Cox, Ross \& Rubestein serão utilizados como benchmark, e que os parâmetros $u, d$ e $q$ definidos neste modelo partem da premissa de que o preço do ativo objeto segue um Movimento Geométrico Browniano (MGB), este processo estocástico será empregado em todas as análises deste trabalho.

Matematicamente, a variação no preço de um ativo financeiro que segue um MGB pode ser definida a partir da seguinte equação diferencial estocástica:

$$
d S=\mu \cdot S \cdot d t+\sigma \cdot S \cdot d z
$$

onde $S$ representa o preço do ativo, $\mu$ representa a sua taxa de retorno esperada, $\sigma$ representa a volatilidade do preço do ativo, e $d z$ representa o processo de Wiener. Este processo considera que o retorno efetivo do ativo é proporcional ao valor de $S$. O mesmo raciocínio é válido para a variância deste processo. Adicionalmente, a equação de simulação deste processo pode ser definida como (para maiores informações, vide Batista, 2007):

$$
S_{t}=S_{0} \cdot \exp \left\{\left[\mu-\left(\sigma^{2} / 2\right)\right] \cdot t+\sigma \cdot z_{t}\right\}
$$

onde, para uma avaliação neutra ao risco, a taxa de retorno esperada do ativo deve ser representada pela taxa de juros livre de risco. 


\section{O MERCADO DE CARBONO E O CÁLCULO DA LINHA DE BASE EM SISTEMAS HIDROTÉRMICOS INTERLIGADOS}

A entrada em vigor do Protocolo de Quioto e as pesadas multas impostas às empresas européias que não conseguirem reduzir as suas emissões dos Gases de Efeito Estufa fazem do mercado de carbono uma realidade na América Latina. De acordo com os estudos realizados pelo Núcleo de Assuntos Estratégicos da Presidência da República (2005), o Brasil se destaca como um dos países de maior potencial para a exportação de RCEs no mundo, em grande parte devido à sua capacidade de produzir energia elétrica a partir de fontes renováveis.

Segundo o estabelecido pelo próprio Protocolo de Quioto (vide UNFCCC), todo projeto desenvolvido no âmbito do Mecanismo de Desenvolvimento Limpo (MDL) deve ter a sua adicionalidade comprovada antes de ser registrado pelo Comitê Executivo das Nações Unidas. Isto significa que, dentre outras coisas, deve ser provado que as emissões de Gases de Efeito Estufa do projeto são inferiores às emissões do seu respectivo cenário de linha de base, que por sua vez pode ser definido como o cenário de emissões que seria observado caso o projeto proposto não fosse implantado.

Neste contexto, motivado pelo forte conservadorismo adotado pelo Comitê Executivo em reconhecer a adicionalidade dos projetos propostos, diversas metodologias têm sido desenvolvidas para a determinação de uma linha de base confiável, destacando-se neste trabalho a utilização da metodologia ACM0002 - Approved Consolidated Methodology n. 2 (2006). Segundo o escopo desta metodologia, o seu principal objetivo é orientar a determinação da linha de base de projetos geradores de energia elétrica a partir de fontes renováveis, sendo exigido que os mesmos estejam conectados à rede elétrica do país onde o projeto será implantado.

Uma vez que a geração de energia elétrica produzida pelo novo projeto (projeto MDL) contribui para a redução de emissões através do deslocamento da energia gerada por usinas já existentes, entende-se que a linha de base do projeto MDL se torna uma função direta do nível de operação destas usinas. Neste caso, nota-se que quanto maior o nível de geração das usinas "pouco emissoras" (por exemplo, usinas hidrelétricas) menor será a linha de base do projeto MDL, entretanto, quanto maior a geração das usinas "muito emissoras" (por exemplo, usinas termoelétricas que se baseiam na queima de combustíveis fósseis) maior será a sua linha de base e, consequentemente, maior será a redução de emissão alcançada pela implantação do projeto MDL.

Note que tal influência pode ser entendida como um fator de risco para o projeto MDL, pois impacta diretamente na quantidade de RCEs a que o mesmo terá direito. Neste contexto, cabe ressaltar que os trabalhos desenvolvidos até o momento não consideram o risco relacionado à capacidade dos projetos MDL produzirem RCEs durante a sua fase operativa. Por exemplo, tanto as análises realizadas pelo Núcleo de Assuntos Estratégicos da Presidência da República (2005), quanto as análises realizadas pela Associação Espanhola da Indústria Elétrica (2005), consideram a linha de base dos projetos MDL constante ao longo do tempo, sendo a mesma determinada com base em dados históricos. Neste trabalho o fator de risco mencionado anteriormente é denominado risco técnico do projeto, o qual é devidamente considerado através da simulação de 2000 cenários para a operação futura das usinas conectadas ao Sistema Interligado Nacional. 
Segundo o escopo da metodologia ACM0002, a linha de base dos projetos de geração de energia elétrica a partir de fontes renováveis, conectados a sistemas elétricos interligados, deve ser determinada por meio da combinação de dois tipos de Fatores de Emissão: o Fator de Emissão da Margem Operacional $\left(E F_{O M}\right)$ e o Fator de Emissão da Margem Construtiva $\left(E F_{B M}\right)$. Este cálculo se encontra representado na equação 18.

$$
E F_{y}=50 \% \cdot E F_{O M, y}+50 \% \cdot E F_{B M, y}
$$

onde $y$ representa o ano no qual a linha de base está sendo calculada. No que tange à determinação de $E F_{O M}$, cabe ressaltar que a metodologia ACM0002 estabelece três diferentes métodos que podem ser aplicados aos projetos desenvolvidos no âmbito do Sistema Interligado Nacional (SIN): o método Simples Ajustado, o método de Análise dos Dados de Despacho, e o método Médio. Tendo em vista que o objetivo deste trabalho não é analisar as características ou comparar os resultados obtidos por meio dos diferentes métodos de cálculo do Fator de Emissão da Linha de Base, mas sim exemplificar a metodologia proposta e verificar a robustez dos métodos de GVW e LSM na avaliação da opção de investimento considerada, apenas o método "Médio" será empregado. A escolha deste método se justifica pela sua simplicidade no cálculo do Fator de Emissão da Margem Operacional.

\subsection{Método Médio}

Este método considera que o cálculo do Fator de Emissão da Margem Operacional deve ser realizado por meio da seguinte equação:

$$
E F_{O M, y}=\left(\sum_{n} G E N_{j, y} \cdot C O E F_{j}\right) / \sum_{n} G E N_{j \cdot y}
$$

onde $G E N_{j, y}$ representa a quantidade de energia elétrica (em MWh) produzida pela usina $j$ durante o ano $y, C O E F_{j}$ representa o coeficiente de emissão de dióxido de carbono (em $\mathrm{tCO}_{2} /$ $\mathrm{MWh}$ ) da fonte primária de energia utilizada pela usina $j$, e $n$ representa o número total de usinas que pertencem ao subsistema onde o projeto MDL está localizado.

Uma vez determinado o Fator de Emissão da Margem Operacional, ressalta-se que o Fator de Emissão da Margem Construtiva deve ser calculado com base na maior geração anual determinada a partir dos seguintes grupos de usinas:

- as últimas cinco usinas construídas no subsistema elétrico do projeto MDL;

- os últimos acréscimos de capacidade do subsistema do projeto que compreendam $20 \%$ da sua geração total.

É válido ressaltar que em ambos os casos todos os projetos MDL previamente construídos devem ser excluídos do cálculo do Fator de Emissão da Margem Construtiva, que por sua vez também pode ser determinado a partir da equação 19. Neste caso, o parâmetro $n$ representará o conjunto de usinas definido por um dos dois grupos definidos anteriormente. 
Finalmente, uma vez que $E F_{O M}$ e $E F_{B M}$ foram determinados, utiliza-se a equação 18 para se determinar o Fator de Emissão da Linha de Base do projeto MDL. Cabe ressaltar que o Fator de Emissão da Linha de Base assim determinado considera que um projeto gerador de energia elétrica a partir de fontes renováveis reduz as emissões de dióxido de carbono ao substituir a energia que é produzida, por meio da queima de combustíveis fósseis, por usinas conectadas ao Sistema Interligado Nacional. Matematicamente, tais reduções podem ser calculadas da seguinte forma:

$$
R E_{y}=E B_{y}-E P_{Y}-F_{y}
$$

onde $R E$ representa a redução das emissões de dióxido de carbono alcançadas devido à operação do projeto MDL, $E B$ representa as emissões correspondentes a sua linha de base, $E P$ representa as emissões do próprio projeto MDL, e $F$ representa as suas fugas ou emissões indiretas do projeto. Ainda na equação 20, ressalta-se que $y$ representa o período de um ano para o qual a atividade do projeto foi monitorada para fins de contabilização das reduções de emissões de dióxido de carbono. Para os empreendimentos analisados neste trabalho, é importante ressaltar que o escopo da metodologia ACM0002 determina que tanto as emissões do projeto MDL quanto as suas respectivas fugas devem ser consideradas nulas. Adicionalmente, as emissões da linha de base devem ser determinadas da seguinte forma:

$$
E B_{y}=E G_{y} \cdot E F_{y}
$$

onde $E G_{y}$ representa a geração de energia elétrica do projeto MDL e $E F_{y}$ representa o seu Fator de Emissão da Linha de Base, ambos determinados para um dado ano $y$.

Uma vez detalhada a metodologia ACM0002, conclui-se que a consideração do risco técnico associado ao desenvolvimento de projetos MDL, conectados a sistemas elétricos interligados, está condicionada ao conhecimento prévio das seguintes variáveis ao longo do período previsto para a sua operação:

- metas de geração térmica e hidráulica do sistema;

- intercâmbios de energia entre os subsistemas elétricos que compõem o sistema interligado do local onde o projeto MDL é desenvolvido;

- a fonte primária de energia utilizada por cada empreendimento do sistema;

- e, finalmente, a configuração da expansão térmica e hidráulica do sistema.

Neste trabalho, cabe ressaltar que tanto a configuração da expansão quanto a fonte primária de energia utilizada pelas usinas do sistema foram obtidos a partir da plataforma de dados considerada pelo Ministério de Minas e Energia do Brasil para a elaboração do Plano Decenal de Expansão de Energia Elétrica (PDEE) 2006-2015 (vide Ministério de Minas e Energia, 2006). É importante ressaltar que as análises do PDEE 2006-2015 são orientadas pelas diretrizes de longo prazo do setor elétrico brasileiro, sendo as mesmas responsáveis por identificar as principais linhas de desenvolvimento dos sistemas elétricos de geração e de transmissão para o Brasil. Tais análises levam em conta os diferentes cenários para o crescimento da economia, para o consumo de energia, de disponibilidade das fontes de geração, de políticas de aumento da eficiência energética, e de desenvolvimento industrial sustentável. 
A partir da plataforma de dados do PDEE 2006-2015, é possível realizar o planejamento da operação energética do Sistema Interligado Nacional, obtendo-se possíveis cenários de geração hidráulica e térmica, além dos intercâmbios de energia entre os subsistemas do SIN para um horizonte igual a dez anos. Desta forma, a incerteza técnica associada à produção de RCEs estará sendo devidamente considerada ao longo do período operacional do projeto MDL, uma vez que diferentes possíveis cenários de linha de base estarão sendo associados à sua operação. Finalmente, vale ressaltar que, neste trabalho, tal análise será realizada utilizando-se o modelo computacional NEWAVE (vide Maceira, 2008), desenvolvido pelo CEPEL, e oficialmente utilizado pelo Operador Nacional do Sistema Elétrico (ONS) para a realização do planejamento da operação energética no Brasil.

\section{ABORDAGEM METODOLÓGICA PROPOSTA}

Neste trabalho, a abordagem metodológica proposta tem como principal característica a união de conceitos e metodologias, usualmente empregadas em diferentes áreas de conhecimento, em uma única abordagem capaz de estimar o valor incremental do mercado de carbono para os projetos de geração de energia elétrica a partir de fontes renováveis. Para tanto, os conceitos e técnicas empregados para o cálculo da linha de base de determinados tipos de projetos MDL, para a realização do planejamento da operação energética em sistemas hidrotérmicos interligados, e para a análise de investimentos sob incerteza são utilizados neste trabalho. A Figura 1 ilustra a abordagem metodológica proposta.

Uma vez conhecidas as características técnicas e econômicas tanto do projeto MDL quanto do Sistema Interligado onde o projeto será implantado, o primeiro passo consiste em gerar possíveis cenários hidrológicos de despacho para as usinas do sistema dentro do horizonte de estudo considerado. Cabe ressaltar que tais cenários são obtidos através da utilização do modelo NEWAVE aplicado à base de dados do Plano Decenal de Expansão de Energia Elétrica 2006-2015.

Uma vez que os cenários hidrológicos são determinados, as duas próximas etapas consistem em se estimar, para cada cenário hidrológico previamente definido, os Fatores de Emissão das Margens Operacional e Construtiva do subsistema elétrico hospedeiro do projeto. Tais fatores são determinados segundo os critérios estabelecidos pela metodologia ACM0002. Após a determinação destes fatores de emissão, o passo seguinte consiste em determinar cenários para o Fator de Emissão da Linha de Base do projeto MDL. Para tanto, utiliza-se a equação 18 definida na Seção 3. É importante destacar que, ao final desta etapa, considera-se que o risco relacionado ao total de RCEs que serão alcançadas pelo projeto se encontra devidamente representado por meio de 2000 cenários que modelam a evolução da sua linha de base.

A próxima etapa consiste em se determinar o valor incremental do mercado de carbono para o projeto considerado. Uma vez que o investidor possui o direito, mas não o dever de realizar um investimento adicional para registrar o seu projeto no Comitê Executivo das Nações Unidas, foi considerado que o detentor do projeto, na verdade, possui uma opção gerencial. Tal opção possibilita ao investidor requerer e comercializar RCEs ao longo da fase operacional do projeto. Neste trabalho, considera-se que os métodos Binomial, GVW e LSM podem ser empregados para avaliar a opção considerada. Ainda é importante observar que o valor da opção é deter- 


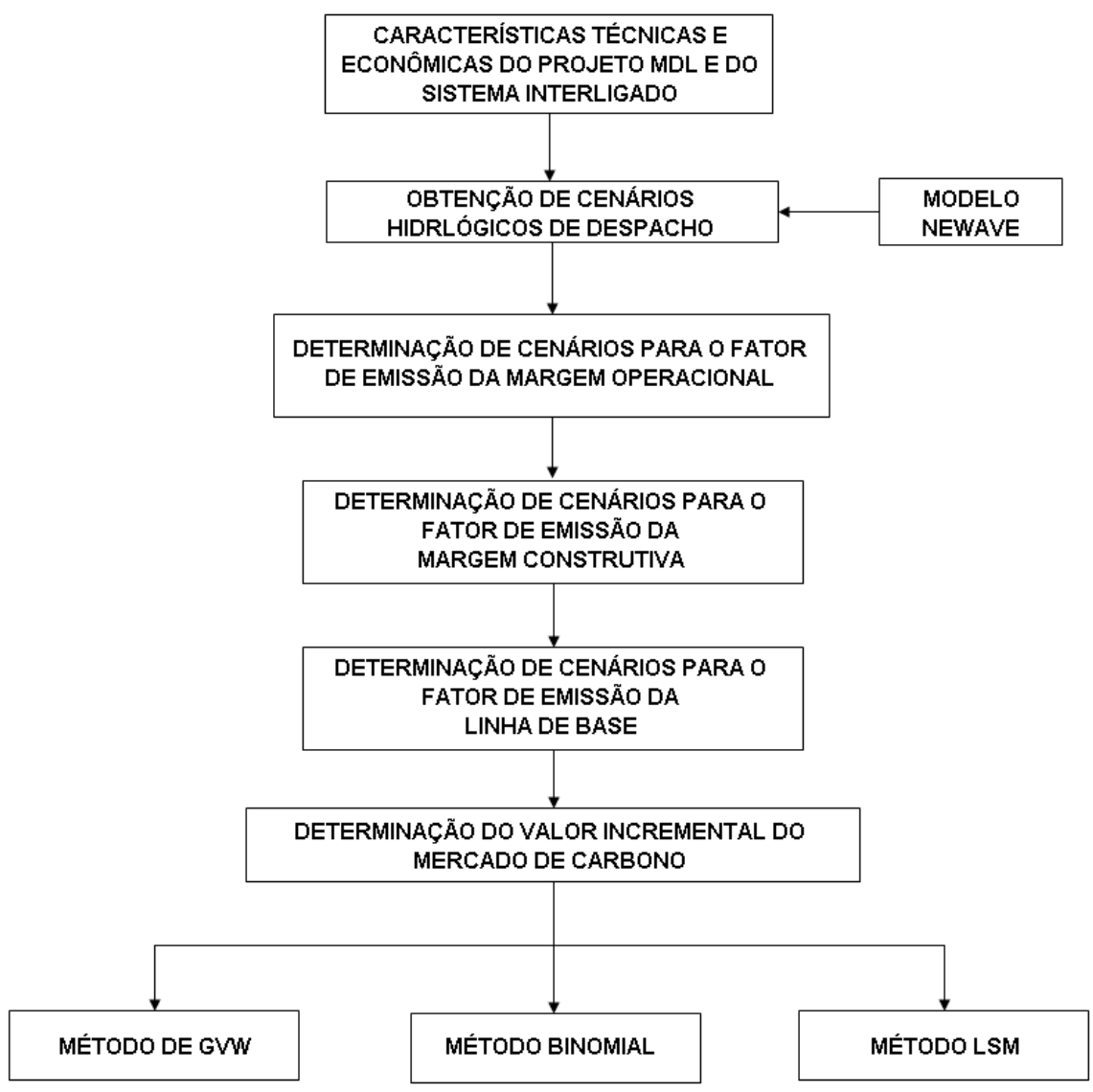

Figura 1 - Abordagem metodológica proposta.

minado para cada um dos cenários de linha de base previamente estimados, sendo que a média destes valores representa o valor final da opção avaliada.

Finalmente, é importante ressaltar que a consideração tanto do risco técnico, associado à linha de base do projeto MDL, quanto do risco de mercado, associado à aleatoriedade dos preços das RCEs, constituem uma inovação na análise de projetos de geração de energia elétrica desenvolvidos no âmbito do Mecanismo de Desenvolvimento Limpo do Protocolo de Quioto.

\section{RESULTADOS}

Nesta seção serão apresentados os resultados de uma investigação sistemática sobre a robustez dos métodos LSM e de GVW quando aplicados na determinação do valor incremental do mercado de carbono nos projetos de geração de energia elétrica no Brasil. Conforme mencionado na Seção 2.1, o método binomial será utilizado como benchmark para verificar a convergência dosresultados encontrados. 
Cabe ressaltar que as análise desta seção serão realizadas tomando por base as características de um empreendimento eólico de $130 \mathrm{MW}$ de capacidade instalada. Considera-se que este empreendimento será implantado no subsistema Sudeste do Sistema Interligado Nacional. Além disso, conforme mencionado na Seção 3 deste trabalho, o método Médio será empregado no cálculo do Fator de Emissão da Margem Operacional do projeto. Considera-se que uma RCE esteja avaliada em US\$ 5,00/ $\mathrm{tCO}_{2}$ e no mercado internacional de carbono. Os demais parâmetros desta análise estarão de acordo com os valores descritos na Tabela 1.

Tabela 1 - Parâmetros do estudo de caso.

\begin{tabular}{|l|c|c|}
\hline \multicolumn{1}{|c|}{ Parâmetro } & Unidade & Valor \\
\hline Período de construção & meses & 18 \\
Vida útil & anos & 20 \\
Custos de transação & US\$ & $137.500,00$ \\
Período de obtenção de RCEs & anos & 10 \\
Custo do capital próprio & $\%$ a.a. & 12,00 \\
Taxa de câmbio & R\$/US\$ & 2,20 \\
Capacidade instalada & MW & 135,00 \\
Fator de capacidade & $\%$ & 23,49 \\
Custo de verificação/Certificação das RCEs & US\$ & $9.000,00$ \\
Custo anual de expedição das RCEs & $\%$ & 11,00 \\
PIS & $\%$ & 1,65 \\
COFINS & $\%$ & 7,60 \\
Alíquota de Imposto de Renda & $\%$ & 25,00 \\
Contribuição Social - CSSL & $\%$ & 9,00 \\
\hline
\end{tabular}

Adicionalmente, considera-se que a receita obtida com a venda das RCEs deverá será calculada da seguinte forma:

$$
R(t)=P \cdot Q(t) \cdot(1-C E C)-C F
$$

onde $R(t)$ representa a receita bruta em um determinado ano $t, P$ representa o preço da RCE no momento do exercício da opção, $Q(t)$ representa a quantidade de RCEs gerada pelo projeto no ano $t, C E C$ representa o percentual de gastos com os processos de expedição e comercialização das RCEs, e $C F$ representa os custos anuais com as etapas de Verificação e Certificação das RCEs. Finalmente, vale ressaltar que a receita auferida pelo projeto com a venda de RCEs ainda será tributada pelo PIS, COFINS, Imposto de Renda e Contribuição Social.

Uma vez que os resultados do método binomial serão utilizados como benchmark nestas análises, é necessário verificar a convergência deste método no sentido de se determinar empiricamente um valor adequado para representar o verdadeiro valor da opção considerada. Neste caso, a medida de precisão utilizada será a raiz do erro quadrático médio percentual do estimador (RMSE), cuja fórmula de cálculo é descrita pela equação 23 a seguir:

$$
C V(\bar{C})=\left[R M S E(\bar{C}) / C_{R E F}\right] \cdot 100
$$


Nesta equação, $C V$ representa o coeficiente de variação ou o RMSE percentual do estimador $\bar{C}$ e $C_{R E F}$ representa o valor verdadeiro (ou de referência) da variável estimada. Finalmente, é importante ressaltar que diversos cenários hidrológicos serão considerados para modelar a incerteza técnica do projeto MDL, sendo que o valor da opção será igual à média dos valores calculados individualmente para cada um destes cenários.

Uma vez que os resultados desta seção consideram que a dinâmica do preço da RCE segue o Movimento Geométrico Browniano, é necessária a definição de alguns parâmetros. Neste caso, considera-se a taxa de juros livre de risco igual a $8 \%$ a.a., a volatilidade anual dos preços da RCE igual a $40 \%$, o dividend yield do ativo objeto igual a 5\% a.a., e o tempo de vida da opção igual a 18 meses. O comportamento do valor da opção em função do número de passos empregados na árvore binomial se encontra representado na Figura 2.

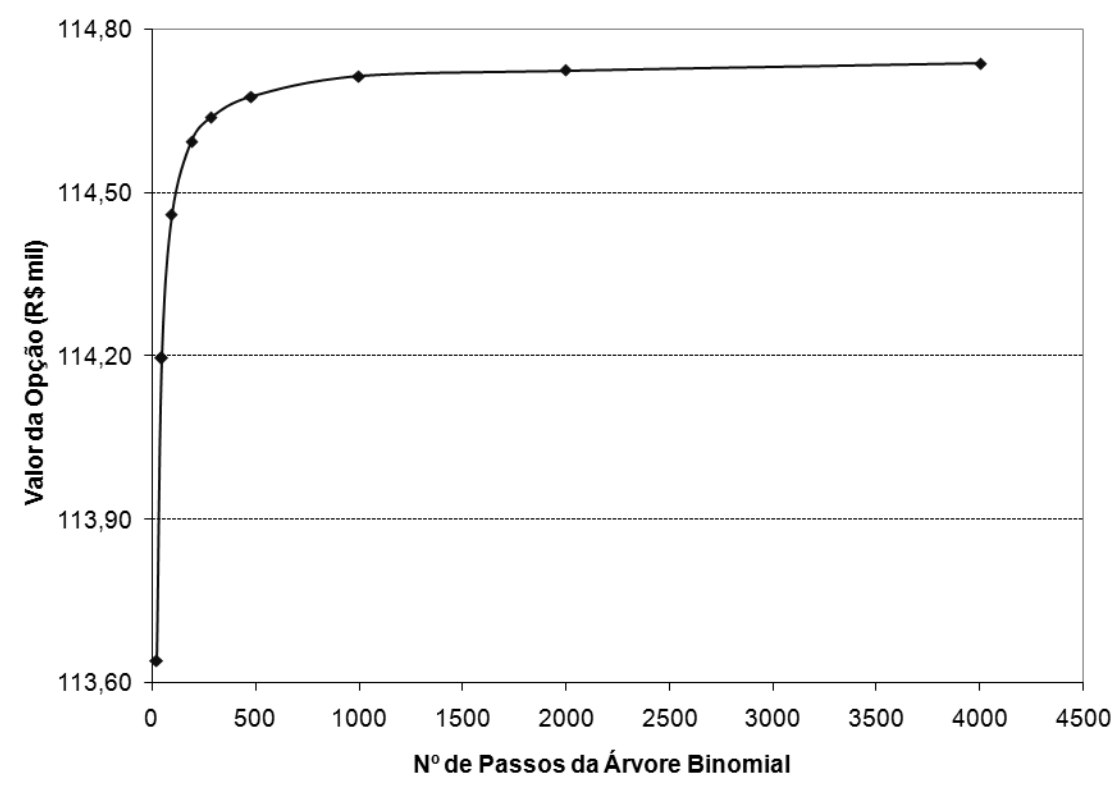

Figura 2 - Valor da opção $\times$ número de passos da árvore binomial.

A partir da Figura 2 conclui-se que o valor da opção converge para um valor próximo a R\$ 114,74 mil à medida que se aumenta o número de passos da árvore binomial. Estendendo esta análise e considerando que, para cada cenário de incerteza técnica, os respectivos valores da opção são especificados pela utilização do método binomial com 4000 passos, é possível avaliar individualmente a convergência do método binomial em cada um destes cenários. A medida de precisão utilizada nesta análise é o viés percentual do estimador, ou seja:

$$
\text { Viés }(\bar{C})=\left[\left(\bar{C}-C_{R E F}\right) / C_{R E F}\right] \times 100
$$

Nesta equação, note que $\bar{C}$ e $C_{R E F}$ possuem o mesmo significado definido na equação 23 . Os resultados desta análise são apresentados na Tabela 2. 
Os resultados da Tabela 2 indicam, dentre todos os cenários de incerteza técnica, o viés da estimativa de menor precisão em função do número de passos empregado na solução do método binomial. Por exemplo, utilizando o método binomial com 2000 passos, nota-se que a estimativa de menor precisão (ou de maior viés) apresenta um viés de $0,12 \%$ em relação ao valor de referência do seu respectivo cenário de incerteza técnica. Em todos os demais cenários de incerteza técnica, a estimativa do valor da opção apresentou um viés inferior a $0,12 \%$.

Tabela 2 - Viés das estimativas realizadas pelo método binomial.

\begin{tabular}{|c|c|c|c|c|c|c|c|c|c|c|}
\hline No. de passos & 12 & 24 & 48 & 96 & 192 & 288 & 480 & 1.000 & 2.000 & 4.000 \\
\hline Viés máximo & $26,83 \%$ & $16,20 \%$ & $8,56 \%$ & $3,94 \%$ & $1,97 \%$ & $1,41 \%$ & $0,75 \%$ & $0,30 \%$ & $0,12 \%$ & $0,00 \%$ \\
\hline
\end{tabular}

Neste contexto, é importante observar a particularidade da opção avaliada neste trabalho em relação às opções financeiras ou reais usualmente analisadas pela literatura acadêmica. Considerando uma opção de investimento sobre um determinado projeto $(V)$, o qual produz um determinado output $(S)$, nota-se a existência de linearidade entre os valores de $V$ e $S$, quando $S$ é modelado segundo determinados processos estocásticos. Por exemplo, supondo $V=q \cdot S$, onde $q$ representa o número de RCEs produzidas ao longo de um determinado período, e $S$ representa o preço da RCE modelado por um MGB, a partir da equação 16 tem-se que:

$$
\begin{aligned}
& d S=\mu \cdot S \cdot d t+\sigma \cdot S \cdot d z \\
& d(q \cdot S)=\mu \cdot q \cdot S \cdot d t+\sigma \cdot q \cdot S \cdot d z \\
& d V=\mu \cdot V \cdot d t+\sigma \cdot V \cdot d z
\end{aligned}
$$

ou seja, o valor do projeto segue um MGB com os mesmos parâmetros do processo estocástico seguido pelo preço da RCE. Note que, em geral, o parâmetro "q" é considerado constante até a maturidade da opção (vide Brennan \& Schwartz, 1985; Dixit \& Pindyck, 1994; Siegel et al., 1987), entretanto, neste trabalho, o mesmo representa uma função direta do Fator de Emissão da Linha de Base do projeto MDL, e, conseqüentemente, pode variar ao longo do tempo.

Supondo que o exercício antecipado da opção seja ótimo em um dado instante $t$ qualquer, nota-se que o Fator de Emissão da Linha de Base será determinado com base nas projeções, ao longo do tempo de operação do projeto, para os montantes de geração térmica e hidráulica no Sistema Interligado Nacional. Uma vez que a proporção entre estes montantes tende a variar ao longo tempo, observa-se que, em um dado cenário de incerteza técnica, o parâmetro $q$ também se tornará variável de acordo com o instante considerado para o exercício da opção. Essa consideração afeta diretamente a relação entre os valores de $V$ e $S$, a qual deixa de ser constante ao longo do tempo em que a opção se mantém "viva".

Finalmente, a partir das análises realizadas nesta seção, optou-se pela utilização dos resultados do método binomial com 4000 passos como benchmark para a verificação da robustez dos métodos LSM e de GVW na determinação do valor incremental do mercado de carbono nos projetos de geração de energia elétrica no Brasil. 


\subsection{Análise de convergência do método de GVW}

Nesta seção, a convergência do método de GVW será verificada quando o mesmo é empregado sob as condições específicas deste trabalho. A medida de precisão adotada será o coeficiente de variação da estimativa, tal como descrito na equação 23. Neste caso, os valores de referência empregados serão os valores obtidos pela aplicação do método binomial com 4000 passos.

Uma vez que o método de GVW utiliza a Simulação de Monte Carlo para determinar o valor de continuação da opção, é importante ressaltar que o custo computacional deste método é bastante elevado quando comparado ao método binomial ou LSM (vide Batista, 2007). Por este motivo, apenas 10 cenários de incerteza técnica foram utilizadas nestas análises.

Assim como o realizado anteriormente, nesta seção o valor da opção também será calculado individualmente para cada cenário de incerteza técnica. Além disso, o coeficiente de variação da estimativa de menor precisão será a variável observada. Os resultados encontrados são apresentados na Tabela 3.

Tabela 3 - Coeficientes de variação das estimativas de menor precisão obtidas pelo método de GVW.

\begin{tabular}{|c|c|c|c|c|}
\hline \multirow{2}{*}{$\begin{array}{c}\text { No. de trajetórias } \\
\text { simuladas }\end{array}$} & \multicolumn{4}{|c|}{ Número de datas de exercício antecipado } \\
\cline { 2 - 5 } & 12 & 24 & 48 & 96 \\
\hline 5.000 & $6,64 \%$ & $11,32 \%$ & $12,38 \%$ & $10,60 \%$ \\
10.000 & $8,99 \%$ & $7,60 \%$ & $8,61 \%$ & $4,68 \%$ \\
20.000 & $10,43 \%$ & $6,48 \%$ & $4,06 \%$ & $3,32 \%$ \\
40.000 & $10,02 \%$ & $6,29 \%$ & $3,50 \%$ & $2,39 \%$ \\
\hline
\end{tabular}

Os resultados da Tabela 3 indicam que o coeficiente de variação máximo dentre as estimativas tende a diminuir à medida que se aumenta o número de trajetórias simuladas para o preço da RCE, sendo que o mesmo comportamento é observado à medida que se aumenta o número de datas onde é possível o exercício antecipado da opção. Por exemplo, considerando 96 datas de exercício antecipado e 40.000 trajetórias simuladas a cada iteração do método de GVW, pode-se dizer que, em todos os cenários de incerteza técnica, os valores estimados possuem coeficiente de variação menor ou igual a 2,39\%.

A partir destes resultados conclui-se que, à medida que se aumenta o número de trajetórias simuladas e o número de datas de exercício antecipado, o método de GVW pode ser considerado robusto para a avaliação de opções com as características consideradas neste trabalho.

\subsection{Análise de convergência do método LSM}

Conforme citado na Seção 2.3, uma das principais premissas do método LSM é supor que a função de continuação da opção pode ser representada por uma combinação linear de funções base. Segundo Longstaff \& Schwartz (2001) diversos tipos de funções podem ser utilizadas com este fim, por exemplo, os polinômios de Laguerre, de Legendre, de Chebyshev ou mesmo os polinômios de Jacobi. 
Neste trabalho utilizou-se o mesmo tipo de função base originalmente empregado por Longstaff \& Schwartz, ou seja:

$$
B_{l}(S)=S^{l}, \quad l=1,2,3, \ldots
$$

onde $S$ representa o preço do ativo objeto e $l$ representa o termo da função de continuação correspondente à respectiva função base. Cabe ressaltar que Longstaff \& Schwartz utilizam a combinação linear de duas funções base para aproximar o valor de continuação $\left(F_{G}(w, t)\right)$ da opção, ou seja,

$$
F_{G}(w, t)=\sum_{l=0}^{G} a_{l} B_{l}(S)=\sum_{l=0}^{2} a_{l} B_{l}(S)=a_{0}+a_{1} \cdot S+a_{2} \cdot S^{2}
$$

onde $G$ representa o número de funções base utilizado e $\alpha$ representa o coeficiente de cada termo de $F_{G}(w, t)$.

Uma vez definida a função de continuação, a mesma oportunidade de investimento anteriormente descrita no início desta seção será utilizada para verificar a robustez do método LSM na determinação do valor incremental do mercado de carbono de projetos de geração de energia elétrica. Novamente, o valor da opção será estimado individualmente para cada cenário de incerteza técnica, sendo que o coeficiente de variação da estimativa de menor precisão será a variável inicialmente observada. Cabe ressaltar que, uma vez que o esforço computacional do método LSM tende a ser menor em relação ao método de GVW, todos os 2000 cenários de incerteza técnica foram considerados nesta análise. Os resultados encontrados são apresentados na Tabela 4. Novamente, os resultados obtidos pelo método Binomial com 4000 passos foram utilizados como benchmark.

Tabela 4 - Coeficientes de variação das estimativas de menor precisão obtidas pelo método de LSM.

\begin{tabular}{|c|c|c|c|c|}
\hline \multirow{2}{*}{$\begin{array}{c}\text { No. de trajetórias } \\
\text { simuladas }\end{array}$} & \multicolumn{4}{|c|}{ Número de datas de exercício antecipado } \\
\cline { 2 - 5 } & 12 & 24 & 48 & 96 \\
\hline 5.000 & $23,62 \%$ & $13,99 \%$ & $16,44 \%$ & $16,76 \%$ \\
10.000 & $26,15 \%$ & $12,31 \%$ & $13,29 \%$ & $8,57 \%$ \\
20.000 & $27,10 \%$ & $12,02 \%$ & $6,78 \%$ & $5,02 \%$ \\
40.000 & $26,08 \%$ & $12,08 \%$ & $6,26 \%$ & $4,38 \%$ \\
\hline
\end{tabular}

Assim como os resultados da Tabela 3, estes resultados também indicam que o coeficiente de variação máximo dentre as estimativas realizadas para cada cenário de incerteza técnica tende a diminuir à medida que se aumenta o número de trajetórias simuladas para o preço da RCE. $\mathrm{O}$ mesmo comportamento também é observado à medida que se aumenta o número de datas onde é possível o exercício antecipado da opção. Por exemplo, considerando 96 datas de exercício antecipado e 20.000 trajetórias simuladas, pode-se dizer que em todos os cenários os valores estimados possuem coeficiente de variação menor ou igual a 5,02\%.

A partir dos resultados apresentados conclui-se que, à medida que se aumenta o número de trajetórias simuladas e o número de datas de exercício antecipado, o método LSM, assim como 
o método de GVW, também pode ser considerado robusto para a avaliação de opções com as características descritas neste trabalho.

\section{CONCLUSÕES}

No presente trabalho verificou-se a robustez dos métodos de Grant, Vora \& Weeks e de Longstaff \& Schwartz para se determinar o valor incremental do mercado de carbono nos projetos que utilizam fontes renováveis para a geração de energia elétrica em sistemas hidrotérmicos interligados, tal como o sistema elétrico brasileiro. É importante ressaltar que esta análise ganha relevância à medida que os métodos LSM e de GVW são considerados mais flexíveis do que o método binomial, o que possibilita a realização de análises mais completas e complexas do problema considerado.

A metodologia proposta apresentou como principal característica a união, em uma única abordagem, de conceitos e técnicas geralmente empregados para o cálculo da linha de base de determinados tipos de projetos MDL, para a realização do planejamento da operação energética em sistemas hidrotérmicos interligados, e para a análise de investimentos sob incerteza.

Uma das principais contribuições da metodologia proposta consiste em determinar a linha de base do projeto MDL de forma prospectiva, levando em consideração o risco associado à sua evolução temporal em cada um dos cenários considerados para a operação do sistema elétrico brasileiro. Tal característica atribui à opção avaliada uma particularidade em relação às opções financeiras ou reais usualmente analisadas, pois, neste caso, o valor do ativo objeto da opção, ou seja, do valor presente das receitas com a venda das RCEs, passa a ser uma função de uma variável estocástica (o preço da RCE) e de um parâmetro cujo valor varia em função do momento ótimo para o exercício da opção.

Outra contribuição deste trabalho é a consideração adicional da aleatoriedade nos preços das Reduções Certificadas de Emissões no desenvolvimento de projetos MDL, considerando assim a ação do gerente ao decidir se vale à pena ou não investir os custos de transação para que o projeto seja registrado no Comitê Executivo das Nações Unidas. A metodologia empregada nesta análise foi a Teoria das Opções Reais.

Utilizando os resultados do método Binomial como benchmark, verificou-se que tanto o método LSM quanto o método de GVW, ambos baseados na técnica de Simulação de Monte Carlo, podem ser considerados robustos para a avaliação de opções com as características descritas neste trabalho. Desta forma, trabalhos futuros envolvendo a utilização de outros processos estocásticos, tais como os processos de reversão à média ou de difusão com saltos, ou ainda a consideração de mais de um fator estocástico, poderão ser realizados em problemas similares.

\section{REFERÊNCIAS}

[1] Asociación Española de LA Indústria ElÉCTRICA. 2005. Metodologías para la Implementación de los Mecanismos flexibles de Kioto - Mecanismo de Desarrollo Limpio en Latinoamérica, Programa Synergy. Disponível em http://www.cordelim.net/extra/html/pdf/library/olade.pdf. 
[2] Baran FD. 2005. Avaliação de uma Floresta de Eucaliptos na Presença de um Mercado de Certificados para Reduções de Emissões de Carbono: Uma Abordagem por Opções Reais. Dissertação de Mestrado, Puc-Rio.

[3] Barros DD. 2006. Modelagem Financeira para Projetos de Tratamento de Resíduos Sólidos no Brasil com base no Mecanismo de Desenvolvimento Limpo do Protocolo de Quioto. Dissertação de Mestrado, COPPEAD/UFRJ.

[4] BATISTA FRS. 2007. Estimação do Valor Incremental do Mercado de Carbono nos Projetos de Fontes Renováveis de Geração de Energia Elétrica no Brasil: Uma Abordagem pela Teoria das Opções Reais. Tese de Doutorado, Departamento de Engenharia Industrial - PUC-Rio.

[5] BRennan MJ \& SChwartz ES. 1985. Evaluating Natural Resource Investments. Journal of Business, 58(2): 135-157.

[6] CAStro AL. 2000. Avaliação de Investimento de Capital em Projetos de Geração Termoelétrica no Setor Elétrico Brasileiro Usando Teoria das Opções Reais. Dissertação de Mestrado, PUC-Rio.

[7] Clean Development Mechanism Executive Board. 2006. Approved Consolidated Baseline Methodology ACM0002: "Consolidated Baseline Methodology for Grid-Connected Electricity From Renewable Sources”, Versão 6, Maio 2006. Disponível em http://cdm.unfccc.int/methodologies/PAmethodologies/approved.html. Último acesso em 05 de Dezembro de 2006.

[8] Cox JC, Ross SA \& Rubinstein M. 1979. Option Pricing: A Simplified Approach. Journal of Financial Economics, 7(3): 229-263.

[9] Dias MAG. 1996. Investimento Sob Incerteza em Exploração \& Produção de Petróleo. Dissertação de Mestrado, Departamento de Engenharia Industrial, PUC-Rio.

[10] Dias MAG. 2005. Opções Híbridas com Aplicações em Petróleo. Tese de Doutorado, PUC-Rio.

[11] DiXIT A \& PINDYCK RS. 1994. Investment under Uncertainty. Princeton University Press, p. $394-$ 405.

[12] Gomes LL. 2002. Avaliação de Termelétricas no Brasil Estudando o Melhor Momento de Investimento por Modelos de Opções Reais. Tese de Doutorado, PUC-RioX

[13] Grant D, Vora G \& Weeks DE. 1996. Simulation and the Early-Exercise Option Problem. The Journal of Finance Engineering, 5(3): 211-227.

[14] Kumbaroglu G et AL. 2004. A Real Options Evaluation Model for the Diffusion Prospects of New Renewable Power Generation Technologies. 6th IAEE European Conference.

[15] Longstaff FA \& Schwartz ES. 2001. Valuing American Options by Simulation: A Simple Least-Squares Approach. The Review of Financial Studies, 14(1): 113-147.

[16] Maceira MeP, Mercio CB \& Gorenstin BG. 1998. Energy Evaluation of The North/Northeastern and South/Southeastern Interconnection with NEWAVE Model. VI SEPOPE, Salvador, Brazil.

[17] Ministério de Minas e Energia. 2006. Plano Decenal de Expansão de Energia Elétrica 20062015. Disponível no site www.mme.gov.br. Último acesso em 07 de Dezembro de 2006.

[18] Núcleo de Estudos Estratégicos da PresidênCia da República. 2005. Cadernos NAE: Mudança do Clima, Volume 2, 2005. Disponível em www.presidencia.gov.br/secom/nae/docs. Último acesso em 01 de Novembro de 2005. 
[19] Ruolz A \& Lasserre P. 2001. La Gestion Optimale d'une forêt exploitée pour son potentiel de diminution des gaz à effet de serre et son bois. Université du Québec à Montréal, Economics Department, Working Paper.

[20] Siddiqui AS, MARnAy C \& Wiser RH. 2005. Real Options Valuation of US Federal Renewable Energy Research, Development, Demonstration and Deployment. 10th Annual Power Research Conference on Eletricity Industry Restructuring.

[21] Siegel DR, Smith JL \& Paddock JL. 1987. Valuing Oil Properties With Option Pricing Models. Midland Corporate Finance Journal, Spring 1987, p. 22-30.

[22] United Nations Framework Convention on Climate Change - UnfCCC. 2005. Protocolo de Quioto, Versão Traduzida pelo Ministério de Ciência e Tecnologia, Brasília. Disponível em www.mct.gov.br/clima/quioto/mdl.htm. Último acesso em 01 de Novembro de 2005. 